\title{
INTERVIEW
}

\section{Radical Technology Meets Radical Application: An Interview with George Church}

\author{
Kevin Davies and George M. Church
}

George Church has been a towering figure in genomics for more than three decades, dating back to the early days of DNA sequencing and the inception of the Human Genome Project. Since then, his interests and influence have spread to systems and synthetic biology, next-gen sequencing technology, and personal and consumer genomics. Church has also had an important role in the launch of CRISPR technology, including a landmark paper in 2013 that marked one of the first demonstrations of CRISPR genome editing in human cells. His group remains among the most creative of research labs, pushing CRISPR to the boundaries of areas including multiplexing technology, de-extinction, xenotransplantation, and gene drives.

Church sat down with Kevin Davies in his office at Harvard Medical School.

Davies: George Church, thanks for the invitation to your office. You're a member of The CRISPR Journal editorial board, let me remind you.

Church: That's right—one of my many conflicts of interest.

Davies: What are some of the key things that are going on in the Church Lab at the moment?

Church: I think the theme is technology developmentradical, enabling, transformative technologies, if we can. Most of them are paired with some really cool applications, a little out of reach with current technologies. We pair radical technology and radical application.

For example, GP-write is something we've been working on for a long time, before it was even named that. The idea is to recode genomes, and the application is to make any organism resistant to all viruses, even viruses we've never seen before. That is recoding, which we're doing from bacteria to mammals, humans, and plants.

Another is organoids. We make organs in pigs by making dozens, maybe 80 different changes in their genome,

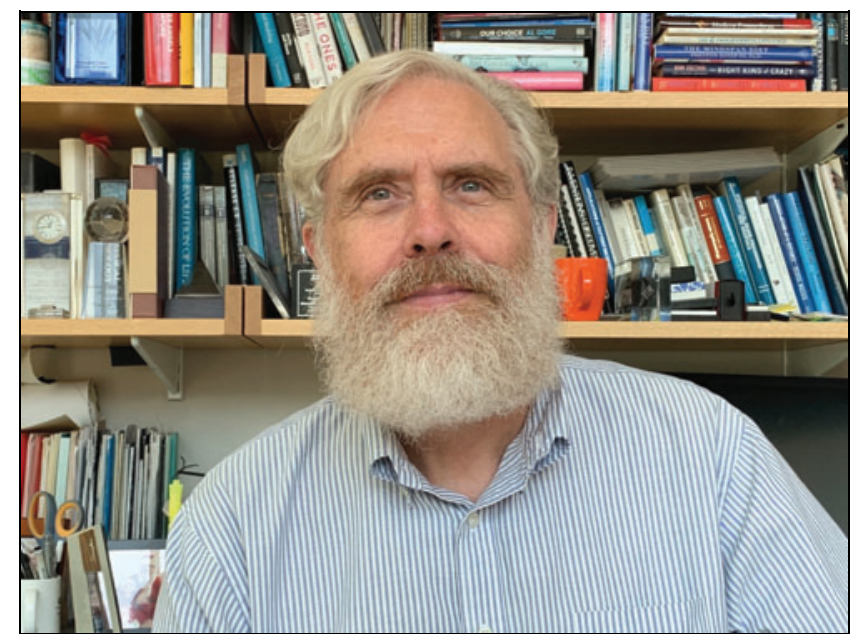

to make them more human-compatible and eliminate their viruses. But we could also make organs from humans. And these are surprising me, how quickly all this is going, and in particular, how you can get definite insights, even diagnostically important insights into very late-onset diseases, such as Alzheimer's, schizophrenia, and bipolar, in about two to four weeks, even though the onset should take 20-70 years. We're making mostly brain organoids, but we have vascularized ones, so we are keen on getting good blood flow so we can make bigger organoids.

We're doing aging reversal. And gene drives, which allow us to cure diseases by dealing with carriers, the animal vectors. We're also looking at other approaches to Lyme disease, including vaccines and people who are exceptionally resistant, analogous to what we've done with human immunodeficiency virus.

Encoding data into DNA. The most recent breakthrough there is that we encoded a terabyte of developmental data into mice. So, every mouse in the colony encodes a fresh terabyte of data, and it only takes a billionth of its body. 
Davies: That's in addition to your book, Regenesis, is it?

Church: That's right. My book was the first encoded in DNA, in 2012. That was half a megabyte. Since then, we've gone from half a megabyte to a terabyte.

Davies: You first got into genome editing before you got into CRISPR, correct?

Church: Oh, way, way before. I got into recombinant DNA in the 1970s-our editing is an honorary member of the recombinant DNA family. I was one of the first employees at Biogen, which used recombinant DNA. As soon as I established my lab in 1986, we had two things: one was reading the genomes, the other was writing genomes. We did homologous recombination, which was precise editing.

Since then, we've gotten grade inflation and distortion of the word "editing." But back in the 1980s, when we started working on editing, it meant doing exactly what you wanted-making exact changes, whether they're big or small. Now, it's like, if you can mangle a gene-I call it genome vandalism-that counts as editing! Most people who are actually editing would be appalled by that definition.

Davies: Before the big CRISPR papers in 2012 and 2013, Feng Zhang spent a period in your lab as a research fellow, working initially on TALENs, right?

Church: Yes, he was a Harvard Junior Fellow. We published six papers together.

Davies: Did your interest in CRISPR begin with Kevin Esvelt?

Church: Probably Prashant Mali. Prashant, Feng, and Kevin all worked together in a little corner in my lab in the Wyss Institute. They were all postdocs, very friendly and very sharing. CRISPR was just on our list of nucleases. There were a bunch of nucleases-Argonaut was another nuclease on our list. But we were always looking for precise editing. And we wanted to do it in normal human cells...

We tried to limit because there are so many things we already do. We wanted to make it harder on ourselves. So, precise editing in human cells was our initial goal, and we did it. The very first paper in January 2013 was precise editing in human cells. And nobody else did normal human cells for quite a while. There's a great temp- tation to use 293 cells, which are quite abnormal, just like HeLa cells or K562. These are all bizarre aneuploidthey are even variable in what their genome looks like. We felt those were completely unacceptable surrogates for clinical applications.

Davies: Did the system that Doudna and Charpentier described in 2012 with the single guide RNA influence your thinking?

Church: That was extremely fundamental. I've said this in the past. CRISPR had been on our list, but Jennifer's work bumped Sp-Cas9 way up on our list at that point. We ended up using a different guide from the one she used ... Feng showed in his Science (2013) paper, ${ }^{1}$ side-by-side with ours, that her guide did not work in his hands.

We didn't make a claim that it didn't work. We just said, "Here is one that does work," and that was the one that we published. It ended up sweeping through. Everybody ended up using that, at least for the Streptococcus pyogenes work.

Davies: In the fall of 2012, you e-mailed Jennifer to say, "Congratulations on a spectacular paper. By the way, we've got this working in human cells."

Church: Right. If I know that I'm converging on somebody's work, I try to check with them. Even though we considered Jennifer a focused biochemist, there was a nonzero probability that she might get interested in human clinical applications. I thought it was courtesy to let her know. I didn't know all the other people who might be working on it.

Keith Joung was part of a NHGRI CEGS grant with me, so I knew he'd be interested. We started the grant, promising to do zinc fingers with Keith, and then we switched to TALENs with Feng, and then CRISPR. We went far beyond what we had promised in the grant.

Davies: You published in January 2013, in the same issue of Science as Feng's paper. A couple of years later, you wrote to The Scientist to correct the narrative that they'd put out in a story because it hadn't mentioned your work and accomplishments in the same context as Feng. Was that a fleeting bit of disgruntlement?

Church: It was just a technical correction. I make such corrections rarely. It just said "three discoverers of the CRISPR-Cas9 system's utility in gene editing." 
But the point is that the first two had not shown editing, and the third one was side by side with ours, and we were the only lab to show editing in normal human cells. I did not want anybody to be left out, but that was it ... It was not me. It was all the postdocs. And not just from my group. I felt that Martin Jinek had been left out of the story, and Prashant Mali and Luhan Yang and Le Cong.

Le Cong was actually a shared student between my lab and Feng Zhang's. He was first author of Feng's 2013 paper. All these people were first authors on these three papers, and you just never heard of them. They were the ones that did a lot of the inspiration, a lot of the work ... I felt there was just this egregious omission.

Davies: You've said that CRISPR-Cas9 almost certainly will not be the last word in gene editing. There's a lot of excitement recently around base editing and, more recently, prime editing. Do you feel fundamentally that is a better way of going about it?

Church: Definitely. We've been involved in the deaminases, the base editors, since before CRISPR ... David Liu's group definitely took it places where we had not gone, and it was fantastic, especially the A-to-G base editor. We pioneer multiplex editing and hene consequent toxicity of CRISPR, whether it is a base editor or notalmost every editor that everybody is using is highly toxic for multiplex editing, which is my obsession, multiplex everything - my middle initial " $M$ "!

So, we did the study where we showed that we could get 26,000 edits. Our previous record was 62 edits in the pig, and we did 26,000 in human cells. And that required scrupulously getting rid of every source of nicks-not just double-stranded breaks, but even nicks that you didn't really think were nicks...

All these damages are extremely toxic in a multiplex environment, which I think is where we are going for a lot of applications. It is just that people talk themselves out of it because it just seems like science fiction. But when you're on an exponential, science fiction becomes science fact while you are blinking. We try to stay a little bit ahead, and so we did this toxicity study, which is now published in bioRxiv. ${ }^{2}$

Davies: We recently heard promising clinical data on Victoria Gray, the first American patient dosed for sickle-cell gene editing ex vivo, and Editas is enrolling patients for their LCA10 trial. What are your hopes for the clinical application of CRISPR over the next few years?

Church: It's great to see gene therapies making it to clinical trials, and even some emerging out of the Phase III trials victorious. Gene therapies hit a major speed bump in 1999-2000. But now we're seeing the field blossom. And it's not just CRISPR, which is mainly subtractive, but also adding a gene that is missing or too low, the latter as we are doing in our aging reversal studies: adding back genes that drop during aging.

My hopes are to lower the expense greatly. I don't want my legacy to be the most expensive drugs in history. We've brought down the price of reading human genomes from $\$ 3$ billion now to $\$ 600$ or even $\$ 0$ with Veritas and Nebula. That I am proud of. I'm much more excited about that than I am about my contribution to expensive therapies.

A key but under-utilized alternative to gene therapy is genetic counseling. If it's really $\$ 0$ for a whole genome sequence now, then as long as we address the privacy and utility issues, everybody could now get their genome sequenced and avoid a huge fraction of these expensive orphan drugs and gene therapies by using genetic counseling instead.

Davies: There was a story about a family in Florida who had a child with spinal muscular atrophy and were refused Zolgensma by their insurance company. They had to crowdsource to raise the money before their infant became ineligible.

Church: I'm not surprised. This is not scalable. What people often forget is that $5 \%$ of births are of this nature. We'd be far better avoiding many of these $\$ 2$ million doses on $5 \%$ of births - by employing preventative genetic counseling. I'm part of a couple of committees that are just looking to estimate the total cost, including opportunity losses and caregiver costs. I estimate it is about $\$ 1$ trillion worldwide per year that we're losing due to serious Mendelian diseases, not to mention all the pain and suffering. That is going to be very hard to sustain...

When people talk about the ethics of CRISPR, $90 \%$ of it should be, and probably is, about equitable distribution of technology. Reducing costs is a major theme of my research. In many cases, reading the genome can be many times more effective.

Davies: As CRISPR expands into the clinic, there seem to be three potential technical concerns. There's the off-target question, the antibody question, and the delivery vehicle. Have those been solved for the most part, or are there still major challenges ahead?

Church: I think the off-target problem has been overstated slightly. I'm partially responsible because, academically, it is a really cool problem that we know how to solve. We and others have published a lot of papers on this. But even in 
the very first papers, we were getting off-targets that were close to the spontaneous mutation rate. If you're going to worry about CRISPR, you should also worry about the spontaneous mutation rate-which is very, very low.

The immune question is hard to evaluate, since I think it is more applicable to CRISPRi and regulatory mechanisms than it is to the hit-and-run of editing. It is once and done-that's the beauty of it. It's not like many drugs, which you have to take for the rest of your life. From that standpoint, the immune question is exaggerated. We're working on ways of making completely human-derived editors, for example human integrases.

I think delivery is definitely the wave of the future. I have two start-ups just launched, one called Dyno and one called Ally, focused on reducing the immune response. Adeno-associated virus already has a very low immune response, but you can take it a bit lower. The side effect of that is that you can get away with lower doses, which brings down the cost and the unintended negative consequences. We're also working on ways that you can target it better. One of the nice things about LCA10 is that you're not dosing the whole body-you're just doing it in the retina. It is the first editing approved for use in vivo.

I would add to your list a fourth issue: CRISPR still does not do its job on target. I think its on-target sins are greater than its off-target sins. The base editors are not perfectly specific for a particularly base, and they're limited to transitions, As to Gs and Cs to Ts. It's very limiting. They're not the Holy Grail, which is precise editing. I think we should hold that concept as our actual goal.

Davies: I look forward to your next company, "Holy Grail"-Grail has already been taken!

Church: Yeah, right!

Davies: Following the CRISPR baby story in 2018, you gave an interview to Science saying you thought there was an element of "piling on" against He Jiankui, bullying perhaps. How do you feel about that now?

Church: I feel the same now, or maybe even more so. I don't think bullying is appropriate. I think careful evaluation of the data is important. I was one of the few people who had access to the data. I felt that people were just saying things as if they knew what was going on. And I felt that that is not very scientific of them.

I got the manuscript from Associated Press (AP). If I'd received it before AP had given it to me, I probably would have felt obliged to point it out to various people, you know, the Food and Drug Administration (FDA), the
National Academy of Sciences (NAS), maybe journalists ... I've also seen it as a reviewer for journals. It's not like he's trying to hide it. But it is not getting published...

I don't want journals to get in the habit of publishing things where there is any doubt about the Institutional Review Board approval or ethics, but in this case, the need to know is high. They made an effort, and the importance of talking about facts rather than speculating outweighs the fact that the approval process was imperfect.

There are many things that were less than desirable here, and this happens again and again-researchers want to jump to the front of the line. They are opportunistic, career oriented, or prize oriented-this happened at the beginning of gene therapy. It's unfortunate because there is much to be done, not just on embryos, but on sperm and egg and so forth. There is a lot that you can do before you have to implant. There is no real law stopping you from doing anything other than implanting.

Davies: Many were surprised at the choice of gene (CCR5) that he targeted because the delta-32 deletion increases risk to West Nile disease and is rarely seen in Asia. Genes are so interconnected, how could we seriously think about tinkering with one gene? Whatever trait you think you are improving, won't there be a slew of unwanted consequences?

Church: Well, welcome to medicine, right? I think what people forget in this discussion, even some of the medical scientists, is that almost every drug that we buy has a long list of small print, and many of them are multigenerational. This is not the first multigenerational medical intervention in history.

For example, smallpox vaccines are still used in the military, and they have a pretty negative consequence $-2 \%$ or something have negative consequences. We did that for many decades ... On the other hand, it is one of the few equitably distributed technologies, where everybody in the world benefits from it, and they no longer have to take the vaccine because the virus is extinct in the wild.

There were no gigantic surprises about $C C R 5$ recently. Most of it was known before. The rules that we converged on at the NAS between 2015 and 2017-the top priority should be bringing in functional variations of the gene that already have precedents in the population. There are lots of people, especially in northern Europe, who are walking around with double nulls in CCR5.

Now, whether they are minus-32 bases or minus-15 bases is probably not a big deal. That is not what people are arguing about so much. But the point is that they are living among us. They may be slightly better in certain categories and slightly worse in others. But it is not 
completely outrageous that you would take one normal person and turn them into a different normal person, right? That happens in medicine all the time.

Davies: You're not against the concept of germline editing? When the technology is ready, and if society gives approval, what might be some of the first genes that would be in the top group of candidates, beyond devastating disease genes?

Church: I'm not even sure about devastating disease genes. I'm not wildly enthusiastic about it; I'm not opposed to it. We should be focusing on outcomes, which is what you're asking about, rather than method. The outcome is that you don't want there to be sickle cell, cystic fibrosis, and thalassemia.

But as I mentioned earlier, most of these can be costeffectively dealt with by genetic counseling. IVF is not pleasant. Whether you're eliminating them by IVF selection or by IVF editing, you still have to do IVF, which is not a pleasant procedure. In fact, many religions would categorize non-implanted embryos as murder. It is neither ethically nor medically pleasant...

Most genetic diseases you could solve in an IVF clinic. If one of the parents is a double-homozygote for a dominant disease that maybe is partially penetrant, and they make it to reproductive age, but they're not sure that their kids will, that would be one example. Infertility is another where there is no other treatment. Germline does have three intrinsic advantages. First, it is better than other delivery systems at reaching all cells in the body. Second, after the first generation, it could be free rather than \$2 million for somatic gene therapy (so like smallpox, an initial investment eventually results in equitable distribution if we work at it). Lastly, germline goes through a single cell, while most somatic therapies impact millions of cells, any one of which could become cancerous. In a single cell, such an event is a priori a million times less likely and, if derived from a clonal set of cells, could be checked by whole-genome sequencing.

Davies: I saw a quote that you gave the Telegraph, where you said, "I just do not think that blue eyes and an extra 15 IQ points is really a public health threat or a threat to our morality." But intelligence is such a polygenic trait-is this even worth discussing?

Church: This is a very interesting point. Many of my colleagues are dismissive that such-and-such is not worth having a discussion about because it is so far out of range. My experience has been things that we did not have to worry about arrive six decades early, right? The affordable genome was supposed to take six decades and arrived in six years!
This is one of those things where I think it's better to worry too much than too little ... Just because it is polygenic doesn't mean it does not have a monogenetic solution. For example, there are seven different clinical indications that are treated with somatotropin, human growth hormone- a single-gene product in the midst of the most complicated genetics of almost any polygenic trait, which is stature...

Furthermore, we're getting better at polygenetic solutions. I mentioned earlier that we can do 26,000 simultaneous edits. That's very different from solving a polygenetic trait, but it is telling us it is going faster than you might think.

Finally, with intelligence in particular, there are a number of mouse experiments that show that two or three genes have an enormous impact on either specific tasks or general tasks that would be categorized as cognitive enhancement. So, does it help putting blinders on or sticking our head in the sand and saying, "There's no way"?

But it is much more likely this is going to be debugged in adults than in the germline because germline takes 20 years of debugging to figure out whether you've got a genius. The market is bigger for adults to become more intelligent and/or reduce cognitive decline, and the development cycle is faster. In principle, you could see an effect in weeks. Adult gene therapies are much scarier to me than germline because you can debug them faster-that will spread like crazy viral memes.

Davies: There are now two international commissions studying germline editing. Is there something that these commissions can do that you think could have some lasting effect? If someone in certain countries is determined to do germline editing, is any learned body or report going to dissuade them?

Church: I'm ready to be pleasantly surprised, but I think it's unlikely that this is going to have a gigantic impact. What will have a gigantic impact are the ongoing experiments that do not involve implantation that will move us forward to greater confidence that we can do things precisely on target and off target and develop things for sperm, egg, and embryo.

For example, you can sequence a clone and determine that it is perfectly on target and nothing off target. And even if you don't do a clonal analysis, what I've never seen anybody mention is that when you dose somebody with gene editing as an adult, or you do it ex vivo-you like to take out their T cells and modify and put them back in-you are dosing 100 million cells. Each one of them is a different CRISPR event, any one of which could hit a tumor suppressor gene, and then it gets amplified, so now it is 
bigger than the original dose of cells. But when you put it in an egg, it is a single event, so your a priori probability of hitting a tumor suppressor exon is a billion times lower. How is that more risky?

There is going to be a lot of technology events that are going to affect things. Also, as Lulu and Nana grow up, every day, their health will be more evident. It's going to be like Louise Brown. It's going to be hard to argue with the fact that they have not died yet, in contrast to the deadly setbacks we had in gene therapy, which did not stop gene therapy.

I wish them well, and I'm happy to put in my two bits. The whole idea that we need a moratorium is crazy, in part because it is often framed as being voluntary. We have (nonvoluntary) penalties for practicing medicine without FDA approval in the United States. Nevertheless, people do it, and the penalties are not that high. If you really want to have an effect, then crank up the penalties; increase whistleblower incentives and increase the surveillance. But you don't need two committees — those things should be obvious.

Davies: You had the cover of Science in 2017 with your company eGenesis and the CRISPR pigs, with 25 endogenous retroviral knockouts. What is the next step?

Church: We're preparing an article describing the rest of the wish list. This is a collection of everybody that has been working on this for more than 20 years. We were latecomers, invited by the pioneers, and we're very indebted to them. When published our first CRISPR article in 2013, and they said, "Oh, that's what we need" because they'd originally hoped it was one or two genes, but then realized it was much more-in the order of 43 genes. We've now engineered in that full wish list.

We've already started primate preclinical trials with a nine-month survival so far. We employ an immunosuppression protocol that is completely compatible with current practice for human-to-human transplants.

Davies: You have a similar company that you are now setting up in China?

Church: Yes, the sibling company called Qihan. They are loosely coupled: Luhan Yang is CEO at Qihan, and $\mathrm{CSO}$ at eGenesis. One notable choice is that we have different strains of pig in the two countries: Bama in China, and Yucatan in America. Some of the advantages of that are not obvious. But one is that there is a strict ban against transporting animal products, living animal pieces, because of foot-and-mouth disease virus and other potential zoonotic diseases. Here, we can prove beyond a shadow of a doubt we did not transfer the living material, just the

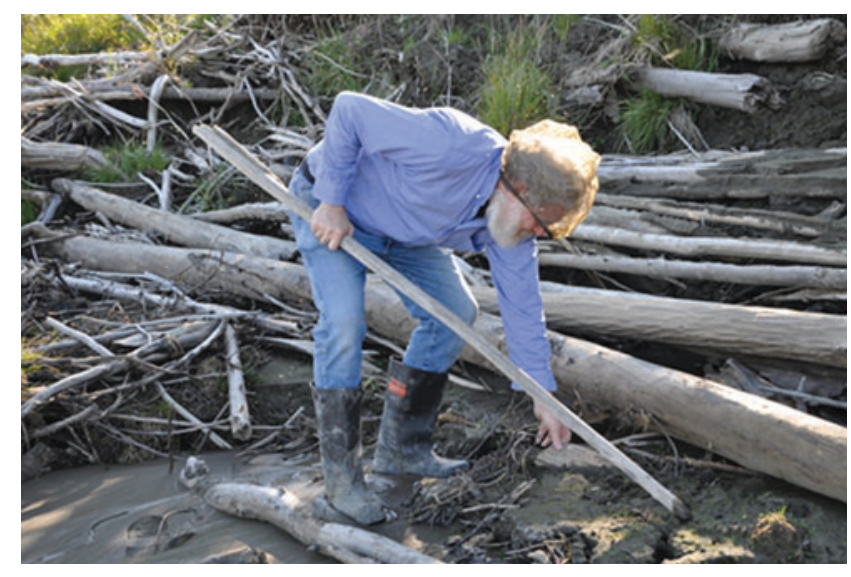

FIG. 1. George Church hunts for mammoth fossils while fending off mosquitos in Pleistocene Park, Siberia, during a visit in 2018 (courtesy: Eriona Hysolli).

information. So, we redo everything. Sometimes a different strain is a bit better.

Davies: In your ASGCT 2019 keynote, you joked about being pestered by journalists who want to know about the woolly mammoth project, and you said there's a lot of work coming along soon. You went to Siberia last year. What was that trip like?

Church: I felt it was overdue (Fig. 1). We've had a very collaborative relationship with the Zimovs, who are running the most likely initial location for our cold-resistant elephants if/ when we get them-Pleistocene Park. There are two now: one near Moscow, which is more convenient for wealthy and powerful Russians, and one in Chersky, which is where I went. It's like 50 hours to get there! It's a beautiful experiment and park; they really have established nearly all the megafauna they need (bison, elk, musk ox, etc.). Just missing a huge herbivore that likes knocking down trees.

In addition to this site visit, evaluating how far along they were in preparing the park, I also went to get mammoth specimens to develop a new technology for analyzing genomic structure. All the ancient DNA people say you cannot get long-range structure, and I think you can ... Whenever I get to do experiments with my own hands, I'm a very happy guy. We dissected six mammoths, and then did the paperwork to get the samples back to the United States. We've done some of the experiments on testing. I'm not going to spoil that story but can say that we're very excited about every aspect of the Siberia trip.

\section{References}

1. Cong $L$, Ran FA, Cox D, et al. Multiplex genome engineering using CRISPR/ Cas systems. Science 2013;339:819-823. DOI: 10.1126/science.1231143.

2. Smith CJ, Castanon O, Said K, et al. Enabling large-scale genome editing by reducing DNA nicking. bioRxiv 2019. DOI: https://doi.org/10.1101/574020 